T. Kurokawa

Nagoya Math. J.

Vol. 111 (1988), 1-11

\title{
INTEGRAL REPRESENTATION OF SMOOTH FUNCTIONS IN WEIGHT CLASSES AND ITS APPLICATION
}

\author{
TAKAHIDE KUROKAWA
}

\section{§. Introduction}

Let $\boldsymbol{R}^{n}$ be the $n$-dimensional Euclidean space, and for each point $x=$ $\left(x_{1}, \cdots, x_{n}\right)$ we write $|x|=\left(x_{1}^{2}+\cdots+x_{n}^{2}\right)^{1 / 2}$. For a multi-index $\alpha=\left(\alpha_{1}, \cdots, \alpha_{n}\right)$, we denote by $x^{\alpha}$ the monomial $x_{1}^{\alpha_{1}} \cdots x_{n}^{\alpha_{n}}$, which has degree $|\alpha|=\sum_{j=1}^{n} \alpha_{j}$. Similarly, if $D_{j}=\partial / \partial x_{j}$ for $1 \leqq j \leqq n$, then

$$
D^{\alpha}=D_{1}^{\alpha_{1}} \cdots D_{n}^{\alpha_{n}}
$$

denotes a differential operator of order $|\alpha|$. We also write $\alpha !=\alpha_{1} ! \cdots \alpha_{n} !$. Throughout this paper, let $1<p<\infty$ and $(1 / p)+\left(1 / p^{\prime}\right)=1$. For a real number $r$, we denote by $L^{p, r}$ the class of all measurable functions $f$ for which

$$
\|f\|_{p, r}=\left(\int\left|f(x)(1+|x|)^{r}\right|^{p} d x\right)^{1 / p}<\infty .
$$

The notation $\mathscr{D}$ denotes the $L F$-space consisting of all $C^{\infty}$-functions with compact support. The symbol $\mathscr{D}^{\prime}$ stands for the topological dual of $\mathscr{D}$. Let $m$ be a positive integer. We denote by $L_{m}^{p, r}$ the space of all $u \in \mathscr{D}^{\prime}$ such that $D^{\alpha} u \in L^{p, r}$ for any $\alpha$ with $|\alpha|=m$. We set

$$
|u|_{m ; p, r}=\sum_{|\alpha|=m}\left\|D^{\alpha} u\right\|_{p, r}
$$

If $u$ belongs to $\mathscr{D}$, then $u$ can be represented by its partial derivatives of $m$-th order as follows (Yu.G. Reshetnyak [4]):

$$
u(x)=\sum_{|\alpha|=m} \frac{m}{\sigma_{n} \alpha !} \int \frac{(x-y)^{\alpha}}{|x-y|^{n}} D^{\alpha} u(y) d y,
$$

where $\sigma_{n}$ denotes the surface area of the unit sphere. In this paper, we are concerned with integral representation of $u \in C^{\infty} \cap L_{m}^{p, r}$ and its integral

Received July 13, 1985.

Revised May 29, 1987. 
estimates. As an application we give an improvement of T.S. Pigolkina's result ([3]).

Throughout this paper, we use the symbol $C$ for generic positive constant whose value may be different at each occurrence, even on the same line.

\section{$\S 2$. Integral representation of smooth functions and its integral estimates}

The following lemma is due to G.O. Okikiolu ([2]).

Lemma 2.1. Let $\left(S, m_{S}\right)$ and $\left(T, m_{T}\right)$ be measure spaces, and let $K(s, t)$ be a measurable function on $S \times T$. Suppose that there are positive measurable functions $\phi_{1}$ on $S, \phi_{2}$ on $T$ and positive constants $M_{1}, M_{2}$ such that

$$
\begin{aligned}
& \int_{T} \phi_{2}(t)^{p^{\prime}}|K(s, t)| d m_{T}(t) \leqq M_{1}^{p^{\prime}} \phi_{1}(s)^{p^{\prime}}, \\
& \int_{S} \phi_{1}(s)^{p}|K(s, t)| d m_{S}(s) \leqq M_{2}^{p} \phi_{2}(t)^{p} .
\end{aligned}
$$

If the operator $K f$ is defined by

$$
K f(s)=\int_{T} K(s, t) f(t) d m_{T}(t)
$$

then

$$
\left(\int_{S}|K f(s)|^{p} d m_{S}(s)\right)^{1 / p} \leqq M_{1} M_{2}\left(\int_{T}|f(t)|^{p} d m_{T}(t)\right)^{1 / p} .
$$

The following lemma is proved by applications of Lemma 2.1.

Lemma 2.2. Let $f$ be a measurable function on $(1, \infty)$. Then:

(i) If $q<1$ and $\ell>0$, then

$$
\int_{1}^{\infty}\left|\int_{1}^{s}(s-t)^{\ell-1} f(t) d t\right|^{p} s^{(q-\ell) p-1} d s \leqq C \int_{1}^{\infty}|f(t)|^{p} t^{q p-1} d t .
$$

(ii) If $\ell>0$, then

$$
\int_{1}^{\infty}\left|\int_{1}^{s}(s-t)^{\ell-1} f(t) d t\right|^{p}(1+\log s)^{-p} s^{(1-\ell) p-1} d s \leqq C \int_{1}^{\infty}|f(t)|^{p} t^{p-1} d t
$$

(iii) If $q>\ell>0$, then

$$
\int_{1}^{\infty}\left|\int_{s}^{\infty}(t-s)^{\ell-1} f(t) d t\right|^{p} s^{(q-\ell) p-1} d s \leqq C \int_{1}^{\infty}|f(t)|^{p} t^{q p-1} d t
$$


Proof. (i) It suffices to show

$$
\int_{1}^{\infty}\left|\int_{1}^{s} s^{-\ell}(s-t)^{\ell-1} t^{-q p+1} f(t) t^{q p-1} d t\right| s^{q p-1} d s \leqq C \int_{1}^{\infty}|f(t)|^{p} \dot{\iota}^{q p-1} d t .
$$

We take $\left(S, m_{S}\right)=\left((1, \infty), s^{q p-1} d s\right),\left(T, m_{T}\right)=\left((1, \infty), t^{q p-1} d t\right)$ and

$$
K(s, t)=\left\{\begin{array}{cl}
s^{-\dot{\varepsilon}}(s-t)^{\ell-1} t^{-q p+1}, & 1<t<s, \\
0, & 1<s \leqq t .
\end{array}\right.
$$

Since $q<1$, we can choose a number $a$ such that $-1 / p^{\prime}<a<-q+(1 / p)$. For $\phi_{1}(s)=\phi_{2}(s)=s^{a}$, we can show (2.1) and (2.2). Hence we obtain (i) by application of Lemma 2.1 .

(ii) It is enough to show

$$
\int_{1}^{\infty}\left|\int_{1}^{s} s^{-\ell}(s-t)^{\ell-1} t^{-p+1} f(t) t^{p-1} d t\right|^{p}(1+\log s)^{-p} s^{p-1} d s \leqq C \int_{1}^{\infty}|f(t)|^{p} t^{p-1} d t .
$$

We take $\left(S, m_{S}\right)=\left((1, \infty),(1+\log s)^{-p} s^{p-1} d s\right),\left(T, m_{T}\right)=\left((1, \infty), t^{p-1} d t\right)$ and

$$
K(s, t)=\left\{\begin{array}{cl}
s^{-\ell}(s-t)^{\ell-1} t^{-p+1}, & 1<t<s, \\
0, & 1<s \leqq t .
\end{array}\right.
$$

We can show (2.1) and (2.2) for $\phi_{1}(s)=s^{-1 / p^{\prime}}(1+\log s)^{(1-\varepsilon) / p^{\prime}}$ and $\phi_{2}(t)=$ $t^{-1 / p^{\prime}}(1+\log t)^{-\varepsilon / p^{\prime}}$ with $0<\varepsilon<1$.

(iii) It is sufficient to show

$$
\int_{1}^{\infty}\left|\int_{s}^{\infty}(t-s)^{\ell-1} t^{-q+(1 / p)} f(t) d t\right|^{p} s^{(q-\ell) p-1} d s \leqq C \int_{1}^{\infty}|f(t)|^{p} d t .
$$

We take $\left(S, m_{S}\right)=\left((1, \infty), s^{(q-\ell) p-1} d s\right),\left(T, m_{T}\right)=((1, \infty), d t)$ and

$$
K(s, t)=\left\{\begin{array}{cl}
(t-s)^{\ell-1} t^{-q+(1 / p)}, & 1<s<t, \\
0, & 1<t \leqq s .
\end{array}\right.
$$

For $\phi_{1}(s)=s^{(\ell-q) / p^{\prime}}$ and $\phi_{2}(t)=t^{-1 / p p^{\prime}}$, we can show (2.1) and (2.2). We complete the proof of the lemma.

Let $u \in C^{\infty}$. For a nonnegative integer $k$, by Taylor's formula $u$ can be represented as follows:

$$
u(x)=\sum_{\mid \eta ! \leqslant k} \frac{(|x|-1)^{|r|}}{\gamma !}\left(x^{\prime}\right)^{\gamma} D^{\gamma} u\left(x^{\prime}\right)+(k+1) \sum_{|r|=k+1} \int_{1}^{|x|} \frac{(|x|-t)^{k}}{\gamma !}\left(x^{\prime}\right)^{\gamma} D^{\gamma} u\left(t x^{\prime}\right) d t
$$

where $x^{\prime}=x /|x|$. The remainder term in Taylor's formula can be considered as integral representation of a function by its partial derivatives. Using spherical coordinates, Taylor's formula and Lemma 2.2 (i), (ii), we 
obtain

Proposition 2.3. Let $\ell$ and $m$ be positive integers such that $\ell \geqq m$, and $u \in C^{\infty}$.

$$
\begin{aligned}
& \text { (i) If } m-(n / p)-r>0 \text {, then } \\
& \left(\int_{|x| \geqq 1}|u(x)|^{p}|x|^{(r-\ell) p} d x\right)^{1 / p} \\
\leqq & C \sum_{|\beta| \geqq \ell-m}\left(\int_{|\xi|=1}\left|D^{\beta} u(\xi)\right|^{p} d \sigma(\xi)\right)^{1 / p}+C \sum_{|\alpha|=\ell-m+1}\left(\int_{|x| \geqq 1}\left|D^{\alpha} u(x)\right|^{p}|x|^{(r-m+1) p} d x\right)^{1 / p} . \\
& \quad \text { (ii) If } m-(n / p)-r=0, \text { then } \\
& \left(\int_{|x| \geqq 1}|u(x)|^{p}(1+\log |x|)^{-p}|x|^{\mid r-\ell) p} d x\right)^{1 / p} \\
\leqq & C \sum_{|\beta| \geqq \ell-m}\left(\int_{|\xi|=1}\left|D^{\beta} u(\xi)\right|^{p} d \sigma(\xi)\right)^{1 / p}+C \sum_{|\alpha|=\ell-m+1}\left(\int_{|x| \geqq 1}\left|D^{\alpha} u(x)\right|^{p}|x|^{(r-m+1) p} d x\right)^{1 / p} .
\end{aligned}
$$

Now we shall deal with integral representation of $u \in C^{\infty} \cap L_{m}^{p, r}$. For this purpose we prepare two lemmas. We denote by $\Sigma_{n}$ the unit sphere $\left\{\xi \in R^{n} ;|\xi|=1\right\}$, and let $m_{\sigma}(E)$ represent the surface area of $E \subset \Sigma_{n}$.

Lemma 2.4. Let $1-(n / p)-r<0$. If $\Phi=\left\{\phi_{i}\right\}_{i=1, \cdots, n} \subset C^{\infty} \cap L^{p, r}$ is a family of functions such that $D_{i} \phi_{j}=D_{j} \phi_{i}$ for all $i, j=1, \cdots, n$, then for each point $x$ there exists a set $E^{\Phi}(x) \subset \Sigma_{n}$ with $m_{\sigma}\left(\Sigma_{n}-E^{\Phi}(x)\right)=0$, which satisfies the following conditions:

(i ) For $\xi \in E^{\Phi}(x)$

$$
\int_{0}^{\infty}\left|\phi_{i}(x-s \xi)\right| d s<\infty, \quad i=1, \cdots, n
$$

(ii) If we put

$$
\psi(x)=\sum_{i=1}^{n} \xi_{i} \int_{0}^{\infty} \phi_{i}(x-s \xi) d s
$$

then $\psi(x)$ is independent of $\xi \in E^{\Phi}(x)$, and $D_{j} \psi=\phi_{j}(j=1, \cdots, n)$.

(iii) For $\xi \in E^{\oplus}(x)$ and $t>0$

$$
\psi(x-t \xi)=\sum_{i=1}^{n} \xi_{i} \int_{t}^{\infty} \phi_{i}(x-s \xi) d s .
$$

Proof. By the assumption $\phi_{i} \in C^{\infty} \cap L^{p, r}$, we see that

$$
\infty>\left.\int_{|y| \geqq 1}\left|\phi_{i}(x-y)\right|\right|^{p}|y|^{p r} d y=\int_{|\xi|=1} \int_{1}^{\infty}\left|\phi_{i}(x-s \xi)\right|^{p} s^{p r+n-1} d s d \sigma(\xi) .
$$


Hence, if we put $E_{x}^{1}=\cup_{i=1}^{n}\left\{\xi ; \int_{1}^{\infty}\left|\phi_{i}(x-s \xi)\right|{ }^{p} s^{p r+n-1} d s=\infty\right\}$, then $m_{\sigma}\left(E_{x}^{1}\right)=$ 0 . By Hölder's inequality and the condition $1-(n / p)-r<0$, we see that for $\xi \in \Sigma_{n}-E_{x}^{1}$

$$
\int_{0}^{\infty}\left|\phi_{i}(x-s \xi)\right| d s<\infty, \quad i=1, \cdots, n
$$

Furthermore, by the assumption $\phi_{i} \in C^{\infty} \cap L^{p, r}$, we have

$$
\begin{aligned}
\infty & >\int_{|y| \geqq 1}\left|\phi_{i}(x-y)\right|^{p}|y|^{p r} d y \\
& =\int_{1}^{\infty} \int_{0}^{2 \pi} \cdots \int_{0}^{\pi}\left|\phi_{i}(x-s \xi)\right|^{p} s^{p r+n-1}\left(\sin \theta_{1}\right)^{n-2} \cdots\left(\sin \theta_{n-2}\right) d \theta_{1} \cdots d \theta_{n-1} d s
\end{aligned}
$$

where $\xi_{j}=\cos \theta_{j} \prod_{k=1}^{j-1} \sin \theta_{k}(1 \leqq j \leqq n-1)$ and $\xi_{n}=\prod_{k=1}^{n-1} \sin \theta_{k}$. Hence there exist sets $D_{x, j}^{i} \subset D_{j}=[0, \pi] \times \cdots \times \widehat{[0, \pi]} \times \cdots \times[0, \pi] \times[0,2 \pi] \subset R^{n-4}$ $(j=1, \cdots, n-1)$ such that $m_{n-2}\left(D_{x, j}^{i}\right)=0$ and for $\left(\theta_{1}, \cdots, \hat{\theta}_{j}, \cdots, \theta_{n-1}\right) \in$ $D_{j}-D_{x, j}^{i}$

$$
\begin{gathered}
\int_{1}^{\infty} \int_{0}^{\pi}\left|\phi_{i}(x-s \xi)\right|^{p} s^{p r+n-1}\left(\sin \theta_{j}\right)^{n-1-j} d \theta_{j} d s<\infty, \quad j=1, \cdots, n-2, \\
\int_{1}^{\infty} \int_{0}^{2 \pi}\left|\phi_{i}(x-s \xi)\right|^{p} s^{p r+n-1} d \theta_{n-1} d s<\infty
\end{gathered}
$$

where $m_{n-2}$ stands for the $(n-2)$-dimensional Lebesgue measure and the symbol $\sim$ denotes that the $j$-th element is deleted. For each positive number $\varepsilon<\pi / 2$, we put $C_{s, j, \varepsilon}\left(\theta_{j}\right)=x-s \xi, \varepsilon \leqq \theta_{j} \leqq \pi-\varepsilon(j=1, \cdots, n-2)$, and $C_{s, n-1}\left(\theta_{n-1}\right)=x-s \xi, 0 \leqq \theta_{n-1} \leqq 2 \pi$. We shall prove that for $\left(\theta_{1}, \cdots\right.$, $\left.\hat{\theta}_{j}, \cdots, \theta_{n-1}\right) \in D_{j}-D_{x, j}^{i}$

$$
\begin{gathered}
\liminf _{s \rightarrow \infty} \int_{\varepsilon}^{\pi-\varepsilon}\left|\phi_{i}(x-s \xi)\right|\left|\frac{d C_{s, j, \varepsilon}\left(\theta_{j}\right)}{d \theta_{j}}\right| d \theta_{j}=0, \quad j=1, \cdots, n-2, \\
\liminf _{s \rightarrow \infty} \int_{0}^{2 \pi}\left|\phi_{i}(x-s \xi)\right|\left|\frac{d C_{s, n-1}\left(\theta_{n-1}\right)}{d \theta_{n-1}}\right| d \theta_{n-1}=0 .
\end{gathered}
$$

We give the proof of (2.5). We note that

$$
\left|\frac{d C_{s, j \varepsilon}\left(\theta_{j}\right)}{d \theta_{j}}\right|=s\left(\sin \theta_{1}\right) \cdots\left(\sin \theta_{j-1}\right) .
$$

We may assume that $\sin \theta_{1} \cdots \sin \theta_{j-1} \neq 0$. Suppose 


$$
\liminf _{s \rightarrow \infty} \int_{\varepsilon}^{\pi-\varepsilon}\left|\phi_{i}(x-s \xi)\right|\left|\frac{d C_{s, j, s}\left(\theta_{j}\right)}{d \theta_{j}}\right| d \theta_{j}=a>0
$$

Then there exists a number $s_{0}$ such that for $s \geqq s_{0}$

$$
a / 2<\int_{\varepsilon}^{\pi-\varepsilon}\left|\phi_{i}(x-s \xi)\right| s\left(\sin \theta_{1}\right) \cdots\left(\sin \theta_{j-1}\right) d \theta_{j} .
$$

By Hölder's inequality we have

$$
\int_{s_{0}}^{\infty} \int_{\varepsilon}^{\pi-\varepsilon}\left|\phi_{i}(x-s \xi)\right|^{p} s^{p r+n-1}\left(\sin \theta_{j}\right)^{n-1-j} d \theta_{j} d s \geqq C a^{p} \int_{s_{0}}^{\infty} s^{p r+n-1-p} d s=\infty
$$

since $1-(n / p)-r<0$. However this contradicts (2.4). Hence we obtain (2.5). We put $E_{x}^{2, i}=\bigcup_{j=1}^{n-2}\left\{\xi ;\left(\theta_{1}, \cdots, \hat{\theta}_{j}, \cdots, \theta_{n-1}\right) \in D_{x, j}^{i}, \quad 0<\theta_{j}<\pi\right\} \cup$ $\left\{\xi ;\left(\theta_{1}, \cdots, \theta_{n-2}\right) \in D_{x, n-1}^{i}, 0 \leqq \theta_{n-1} \leqq 2 \pi\right\}$, and $E^{\Phi}(x)=\Sigma_{n}-\left(E_{x}^{1} \cup \cup_{i=1}^{n} E_{x}^{2, i}\right)$. Then $m_{\sigma}\left(\Sigma_{n}-E^{\Phi}(x)\right)=0$. By (2.3), (2.5), (2.6) and Stokes' theorem, we see that for $\xi, \eta \in E^{\Phi}(x)$

$$
\sum_{i=1}^{n} \xi_{i} \int_{0}^{\infty} \phi_{i}(x-s \xi) d s=\sum_{i=2}^{n} \eta_{i} \int_{0}^{\infty} \phi_{i}(x-s \eta) d s .
$$

The formulas $D_{j} \psi=\phi_{j}(j=1, \cdots, n)$ follow from Stokes' theorem. Thus we obtain (i) and (ii). The assertion (iii) follows from (ii) and the fundamental theorem of calculus. We complete the proof of the lemma.

By repeating use of Lemmas 2.2 (iii) and 2.4, we have

Lemma 2.5. Let $m-(n / p)-r<0$ and $u \in C^{\infty} \cap L_{m}^{p, r}$. Then:

(i) For a multi-index $\alpha$ with $|\alpha|=m$

$$
\int|x-y|^{m-n}\left|D^{\alpha} u(y)\right| d y<\infty
$$

for every $x \in R^{n}$.

(ii) There exists a set $E_{x} \subset \Sigma_{n}$ with $m_{\sigma}\left(\Sigma_{n}-E_{x}\right)=0$ such that for $\xi \in E_{x}$

$$
\sum_{|\alpha|=m} \int_{0}^{\infty} s^{m-1}\left|D^{\alpha} u(x-s \xi)\right| d s<\infty
$$

and

$$
\sum_{|\alpha|=m}(m / \alpha !) \xi^{\alpha} \int_{0}^{\infty} s^{m-1} D^{\alpha} u(x-s \xi) d s
$$

is independent of $\zeta \in E_{x}$.

(iii) If we set 


$$
v(x)=\sum_{|\alpha|=m}(m / \alpha !) \xi^{\alpha} \int_{0}^{\infty} s^{m-1} D^{\alpha} u(x-s \xi) d s, \quad \xi \in E_{x},
$$

then for $\xi \in E_{x}$ and $t>0$

$$
v(x-t \xi)=\sum_{|\alpha|=m}(m / \alpha !) \xi^{a} \int_{t}^{\infty}(s-t)^{m-1} D^{\alpha} u(x-s \xi) d s .
$$

(iv)

$$
\left(\int_{|x| \geqq 1}|v(x)|^{p}|x|^{p(r-m)} d x\right)^{1 / p} \leqq C \sum_{|\alpha|=m}\left(\int_{|x| \geqq 1}\left|D^{\alpha} u(x)\right|^{p}|x|^{p r} d x\right)^{1 / p} .
$$

Now we shall prove

Theorem 2.6. Let $u \in C^{\infty} \cap L_{m}^{p, r}$ and we suppose that the integral part $[m-(n / p)-r]=k$ of $m-(n / p)-r$ is not greater than $m-2$. Then there exists a polynomial $P(x)=\sum_{k+1 \leqq|\beta| \leqq m-1} c_{\beta} x^{\beta}$ such that, if we set $v(x)=u(x)$ $-P(x)$, then for $k+1 \leqq|\gamma| \leqq m-1$

$$
\begin{aligned}
D^{r} v(x) & =\sum_{|\alpha|=m-|\gamma|} \frac{m-|\gamma|}{\sigma_{n} \alpha !} \int \frac{(x-y)^{\alpha}}{|x-y|^{n}} D^{\alpha+\gamma} u(y) d y \\
& =\sum_{|\alpha|=m-|\gamma|} \frac{m-|\gamma|}{\alpha !} \xi^{\alpha} \int_{0}^{\infty} s^{m-|r|-1} D^{\alpha+\gamma} u(x-s \xi) d s
\end{aligned}
$$

for almost every $\xi \in \Sigma_{n}$.

Proof. For each $\beta$ with $|\beta|=m-1$ we see that

$$
D^{\beta} u\left(-t_{1} \xi\right)-D^{\beta} u(-t \xi)=-\sum_{j=1}^{n} \xi_{j} \int_{t}^{t_{1}} D^{e_{j}+\beta} u(-s \xi) d s
$$

where the symbol $e_{j}$ stands for the multi-index $(0, \cdots, \stackrel{j}{1}, \cdots, 0)$. Since $k \leqq m-2$ implies $1-(n / p)-r<0$, by the condition $u \in C^{\infty} \cap L_{m}^{p, r}$ and Hölder's inequality we have

$$
\int_{0}^{\infty}\left|D^{e_{j}+\beta} u(-s \xi)\right| d s<\infty
$$

for almost every $\xi \in \Sigma_{n}$. Hence $D^{\beta} u\left(-t_{1} \xi\right)$ converges to $C_{\beta}(\xi)$ as $t_{1} \rightarrow \infty$, and by (2.7) we have

$$
C_{\beta}(\xi)-D^{\beta} u(0)=-\sum_{j=1}^{n} \xi_{j} \int_{0}^{\infty} D^{e_{j}+\beta} u(-s \xi) d s
$$

for almost every $\xi \in \Sigma_{n}$. It follows from Lemma 2.5 and (2.8) that $C_{\beta}(\xi)$ are the same for almost all $\xi \in \Sigma_{n}$ and we write $C_{\beta}(\xi)=C_{\beta}$. Moreover we have 


$$
D^{\beta} u(x-t \xi)-D^{\beta} u(x)=-\sum_{j=1}^{n} \xi_{j} \int_{0}^{t} D^{e_{j}+\beta} u(x-s \xi) d s .
$$

By an argument similar to the proof of Lemma 2.4 (ii), we see that $D^{\beta} u(x-t \xi)-D^{\beta} u(-t \xi) \rightarrow 0$ as $t \rightarrow \infty$ for almost every $\xi \in \Sigma_{n}$. Hence for almost every $\xi \in \Sigma_{n}$ we have

$$
D^{\beta} u(x)=C_{\beta}+\sum_{j=1}^{n} \xi_{j} \int_{0}^{\infty} D^{e_{j}+\beta} u(x-s \xi) d s .
$$

We put $u_{1}(x)=u(x)-\sum_{|\beta|=m-1}\left(C_{\beta} / \beta !\right) x^{\beta}$. For $\beta$ with $|\beta|=m-1$ we see that

$$
D^{\beta} u_{1}(x)=D^{\beta} u(x)-C_{\beta}=\sum_{j=1}^{n} \xi_{j} \int_{0}^{\infty} D^{e_{j}+\beta} u(x-s \xi) d s
$$

for almost every $\xi \in \Sigma_{n}$. Next let $2 \leqq \ell \leqq m-k-2$. Suppose that $u_{\ell}(x)$ $=u(x)-\sum_{m-\ell \leqq|\beta| \leqq m-1}\left(C_{\beta} / \beta !\right) x^{\beta}$ and for each multi-index $\gamma$ with $m-\ell \leqq$ $|\gamma| \leqq m-1, D^{\gamma} u_{\ell}$ can be represented as follows:

$$
D^{\gamma} u_{\ell}(x)=\sum_{|\delta|=m-|\gamma|} \frac{m-|\gamma|}{\delta !} \xi^{\delta} \int_{0}^{\infty} s^{m-|\gamma|-1} D^{\delta+r} u(x-s \xi) d s
$$

for almost every $\xi \in \Sigma_{n}$. If $|\gamma| \geqq m-\ell \geqq k+2$, then $m-|\gamma|-(n / p)-r$ $<0$, so that by Lemma 2.5 (iv) we have

$$
\int_{|x| \geqq 1}\left|D^{r} u_{\ell}(x)\right|^{p \mid}|x|^{p(r-(m-|r|))} d x<\infty,
$$

and hence, since $m-|\gamma|-(n / p)-r<-1$,

$$
\int_{0}^{\infty}\left|D^{r} u_{\ell}(-s \xi)\right| d s<\infty
$$

for almost every $\xi \in \Sigma_{n}$. Therefore for $|\zeta|=m-\ell-1, D^{\varsigma} u_{\ell}(-s \xi)$ converges to $C_{\zeta}$ as $s \rightarrow \infty$, and

$$
D^{\zeta} u_{\ell}(x)=C_{\zeta}+\sum_{j=1}^{n} \xi_{j} \int_{0}^{\infty} D^{e_{j}+\zeta} u_{\ell}(x-s \xi) d s
$$

for almost every $\xi \in \Sigma_{n}$. We put $u_{\ell+1}=u_{\ell}(x)-\sum_{|\zeta|=m-\ell-1}\left(C_{\zeta} / \zeta !\right) x^{\zeta}=u(x)$ $-\sum_{m-\ell-1 \leqq|\beta| \leqq m-1}\left(C_{\beta} / \beta !\right) x^{\beta}$. For $\gamma$ with $m-\ell \leqq|\gamma| \leqq m-1$ we see that

$$
D^{\gamma} u_{\ell+1}(x)=D^{\gamma} u_{\ell}(x)=\sum_{|\delta|=m-|r|} \frac{m-|\gamma|}{\delta !} \xi^{\delta} \int_{0}^{\infty} s^{m-|r|-1} D^{\delta+\gamma} u(x-s \xi) d s .
$$

Let $|\gamma|=m-\ell-1$. Since $D^{e_{j}+\gamma} u \in C^{\infty} \cap L^{p, r}$ and $\ell-(n / p)-r<0$, by Lemma 2.8(iii) and Fubini's theorem we have 


$$
\begin{aligned}
D^{r} u_{\ell+1}(x) & =D^{r} u_{\ell}(x)-C_{r}=\sum_{j=1}^{n} \xi_{j} \int_{0}^{\infty} D^{e_{j}+\gamma} u_{\ell}(x-s \xi) d s \\
& =\sum_{j=1}^{n} \xi_{j} \int_{0}^{\infty} \sum_{|\delta|=m-|\gamma|-1} \frac{m-|\gamma|-1}{\delta !} \xi^{\delta} \int_{t}^{\infty}(s-t)^{m-|\gamma|-2} D^{e_{j}+\delta+\gamma} u(x-s \xi) d s d t \\
& =\sum_{|\delta|=m-|\gamma|} \frac{m-|\gamma|}{\delta !} \xi^{\delta} \int_{0}^{\infty} s^{m-|\gamma|-1} D^{\delta+\gamma} u(x-s \xi) d s
\end{aligned}
$$

for almost every $\xi \in \Sigma_{n}$. Thus we obtain the function $v=u_{m-k-1}$ which possesses the following properties: $v(x)=u(x)-\sum_{k+1 \leqq|\beta| \leqq m-1}\left(C_{\beta} / \beta !\right) x^{\beta}$ and for $\gamma$ with $k+1 \leqq|\gamma| \leqq m-1$

$$
D^{\gamma} v(x)=\sum_{|\alpha|=m-|\gamma|} \frac{m-|\gamma|}{\alpha !} \xi^{\alpha} \int_{0}^{\infty} s^{m-|\gamma|-1} D^{\alpha+\gamma} u(x-s \xi) d s
$$

for almost every $\xi \in \Sigma_{n}$. Therefore we also have

$$
\begin{aligned}
D^{r} v(x) & =\left(1 / \sigma_{n}\right) \int_{|\xi|=1} D^{\alpha} v(x) d \sigma(\xi) \\
& =\sum_{|\alpha|=m-|r|} \frac{m-|\gamma|}{\sigma_{n} \alpha !} \int_{|\xi|=1} \int_{0}^{\infty} \xi^{\alpha} s^{m-|\gamma|-1} D^{\alpha+\gamma} u(x-s \xi) d s d \sigma(\xi) \\
& =\sum_{|\alpha|=m-|r|} \frac{m-|\gamma|}{\sigma_{n} \alpha !} \int \frac{(x-y)^{\alpha}}{|x-y|^{n}} D^{\alpha+\gamma} u(y) d y .
\end{aligned}
$$

we complete the proof of the theorem.

The following corollary is a consequence of Proposition 2.3, Lemma 2.5 and Theorem 2.6.

Corollary 2.7. Let $k=[m-(n / p)-r]$ and $u \in C^{\infty} \cap L_{m}^{p, r}$. Then there exists a polynomial $P(x)=\sum_{k+1 \leqq|\beta| \leqq m-1} C_{\beta} x^{\beta}$ such that, if we set $v(x)$ $=u(x)-P(x)$, then for $\gamma$ with $k+1 \leqq|\gamma| \leqq m-1$

$$
\left(\int_{|x| \geqq 1}\left|D^{\gamma} v(x)\right|^{p}|x|^{p(r-(m-|r|))} d x\right)^{1 / p} \leqq C \sum_{|\alpha|=m}\left(\int_{|x| \geqq 1}\left|D^{\alpha} u(x)\right|^{p}|x|^{p r} d x\right)^{1 / p}
$$

and for $\gamma$ with $|\gamma| \leqq k$

$$
\begin{aligned}
& \left(\int_{|x| \geqq 1}\left|D^{\gamma} v(x)\right|^{p}|x|^{p(r-(m-|r|))} d x\right)^{1 / p} \\
& \leqq C \sum_{|\alpha|=m}\left(\int_{|x| \geqq 1}\left|D^{\alpha} u(x)\right|^{p}|x|^{p r} d x\right)^{1 / p}+C \sum_{|r| \leqq|\delta| \leqq n}\left(\int_{|\xi|=1}\left|D^{\delta} v(\xi)\right|^{p} d \sigma(\xi)\right)^{1 / p} \\
& \quad(m-(n / p)-r \neq 0,1, \cdots, m-1) \\
& \left(\int_{|x| \geqq 1}\left|D^{r} v(x)\right|^{p}(1+\log |x|)^{-p}|x|^{p(r-(m-|\gamma|))} d x\right)^{1 / p}
\end{aligned}
$$




$$
\begin{gathered}
\leqq C \sum_{|\alpha|=m}\left(\int_{|x| \geqq 1}\left|D^{\alpha} u(x)\right|^{p}|x|^{p r} d x\right)^{1 / p}+C \sum_{|r| \leqq|\delta| \leqq n}\left(\int_{|\xi|=1}\left|D^{\delta} v(\xi)\right|^{p} d \sigma(\xi)\right)^{1 / p} \\
(m-(n / p)-r=0,1, \cdots, m-1) .
\end{gathered}
$$

\section{§ 3. Density of finite functions}

T.S. Pigolkina [3] proved that, if $r \leqq 0$, then for $u \leqq L_{m}^{p, r}$ there exists a sequence $\left\{\phi_{N}\right\}_{N=1,2, \ldots} \subset \mathscr{D}$ such that $\left|u-\phi_{N}\right|_{m ; p, r}$ tends to 0 as $N \rightarrow \infty$. In this section we show that the above assertion holds for all real number $r$.

First we establish an analogue of Theorem 3.1 in [1]. By [1; p. 13], for each positive integer $m$, there exists a function $\omega_{m}$ which possesses the following properties:

(i) $\omega_{m} \in C^{\infty}$.

(ii) $\omega_{m}(x)=0$ for $x$ with $|x| \geqq 1$.

(iii) $\int \omega_{m}(x) d x=1$.

(iv) $\int \omega_{m}(x) x^{\gamma} d x=0$ for $\gamma$ with $1 \leqq|\gamma| \leqq m-1$.

We put $\Omega_{0}=\{|x|<5 / 4\}$ and $\Omega_{j}=\left\{3\left(2^{j-3}\right)<|x|<9\left(2^{j-3}\right)\right\}, j=1,2, \ldots$. As in $\left[1\right.$; Lemma 1.2], for $\left\{\Omega_{j}\right\}_{j=0,1, \ldots}$ there exist functions $\left\{\rho_{j}\right\}_{j=0,1, \ldots \subset C^{\infty}}$ which satisfy the following conditions:

(i ) $\rho_{j} \geqq 0$ and $\sum_{j=0}^{\infty} \rho_{j}(x)=1$ for all $x \in R^{n}$.

(ii) $\operatorname{supp} \rho_{j} \subset \Omega_{j}, j=0,1, \cdots$.

(iii) $\left|D^{\alpha} \rho_{j}(x)\right| \leqq C 2^{-j|\alpha|}$ for all $x \in R^{n}$.

For a locally integrable function $u$, we set

$$
E_{\varepsilon}^{m} u(x)=\sum_{j=0}^{\infty} \rho_{j}(x) \int u\left(x-\varepsilon 2^{j} y\right) \omega_{m}(y) d y, \quad \varepsilon>0 .
$$

By an argument similar to the proof of Theorem 3.1 in [1], we obtain

Proposition 3.1. If $u \in L_{m}^{p, r}$, then $E_{\varepsilon}^{m} u \in C^{\infty} \cap L_{m}^{p, r}$ and $\left|E_{\varepsilon}^{m} u-u\right|_{m ; p, r}$ tends to 0 as $\varepsilon \rightarrow 0$.

Now we shall prove

THeOREM 3.2. If $u \in L_{m}^{p, r}$, then there exists a sequence $\left\{\phi_{N}\right\}_{N=1,2, \ldots} \subset \mathscr{D}$ such that $\left|u-\phi_{N}\right|_{m ; p, r}$ tends to 0 as $N \rightarrow \infty$.

Proof. By Proposition 3.1, it suffices to show the theorem for $u \in C^{\infty}$ $\cap L_{m}^{p, r}$. By Corollary 2.7 there exists a polynomial $P$ of degree $m-1$ such that, if we put $v=u-P$, then $v$ has the properties in Corollary 
2.7. We take a function $h \in C^{\infty}\left(R^{1}\right)$ such that $0 \leqq h \leqq 1, h(t)=1$ for $t \leqq 1$ and $h(t)=0$ for $t \geqq 2$. For $N=2,3, \cdots$, we set

$$
g_{N}(x)=\left\{\begin{array}{cl}
h((\log |x|)) / \log N), & \text { for } x \neq 0, \\
1, & \text { for } x=0 .
\end{array}\right.
$$

Then $g_{N} \in \mathscr{D}$. For a multi-index $\beta$ with $|\beta| \geqq 1$ we have

$$
\begin{aligned}
& D^{\beta} g_{N}(x)=0 \quad \text { if }|x|<N \text { or if }|x|>N^{2}, \\
& \left|D^{\beta} g_{N}(x)\right| \leqq C(\log N)^{-1}|x|^{-|\beta|} \text { for all } x \in R^{n} .
\end{aligned}
$$

We put $\phi_{N}=g_{N} v$. For $\alpha$ with $|\alpha|=m$ we see that

$\left\|D^{\alpha}\left(u-\phi_{N}\right)\right\|_{p, r}=\left\|D^{\alpha}\left(v-g_{N} v\right)\right\|_{p, r}$

$$
\leqq\left(\int_{|x| \geqq N}\left|D^{\alpha} v(x)\right|^{p}(1+|x|)^{p r} d x\right)^{1 / p}+C \sum_{\beta<\alpha}\left(\int\left|D^{\alpha-\beta} g_{N}(x) D^{\beta} v(x)\right|^{p}(1+|x|)^{p r} d x\right)^{1 / p}
$$

$=I_{N}^{1}+I_{N}^{2}$.

Since $D^{\alpha} v=D^{\alpha} u \in L^{p, r}, I_{N}^{1}$ tends to 0 as $N \rightarrow \infty$. By (3.1) and (3.2) we have

$$
I_{N}^{2} \leqq C \sum_{\beta<\alpha}\left(\int_{N \leqq|x| \leqq N^{2}}\left|D^{\beta} v(x)\right|^{p}(1+\log |x|)^{-p}|x|^{p(r-(m-|\beta|))} d x\right)^{1 / p} .
$$

Hence by Corollary 2.7, $I_{N}^{2}$ tends to 0 as $N \rightarrow \infty$. Since $\phi_{N} \in \mathscr{D}$, we obtain the theorem.

\section{REFERENCES}

[1] V. I. Burenkov, Mollifying operators with variable step and their application to approximation by infinitely differentiable functions, Nonlinear Analysis, Function Spaces and Applications Vol.2, Teubner-texte zur Math., Leipzig, 1982, 5-37.

[2] G. O. Okikiolu, On inequalities for integral operators, Glasgow Math. J., 11 (1970), 126-133.

[ 3 ] T. S. Pigolkina, The density of finite functions in weight classes, Math. Notes, 2 (1967), 518-522.

[4] Yu. G. Reshetnyak, The concept of capacity in the theory of functions with generalized derivatives, Siberian Math. J., 10 (1969), 818-842.

Department of Mathematics

College of Liberal Arts

Kagoshima University

Kagoshima 890, Japan 